\title{
Learning from Mistakes
}

\section{Factors that Influence How Students and Residents Learn from Medical Errors}

\author{
Melissa A. Fischer, MD, MEd, ${ }^{1}$ Kathleen M. Mazor, EdD, ${ }^{2}$ Joann Baril, BS, ${ }^{3}$ Eric Alper, MD, ${ }^{4}$ \\ Deborah DeMarco, MD, ${ }^{5}$ Michele Pugnaire, $M D^{6}$ \\ 'Department of Internal Medicine and Meyers Primary Care Institute, University of Massachusetts Medical School, Worcester, MA, USA; \\ ${ }^{2}$ Meyers Primary Care Institute, University of Massachusetts Medical School Fallon Foundation, and Fallon Community Health Plan, \\ Worcester, MA, USA; ${ }^{3}$ Meyers Primary Care Institute, University of Massachusetts Medical School Fallon Foundation, and Fallon Community \\ Health Plan, Worcester, MA, USA; ${ }^{4}$ Department of Internal Medicine University of Massachusetts Medical School, Worcester, MA, USA; \\ ${ }^{5}$ Department of Internal Medicine, University of Massachusetts Medical School, Worcester, MA, USA; ${ }^{6}$ Office of Medical Education and \\ Department of Family and Community Medicine, University of Massachusetts Medical School, Worcester, MA, USA.
}

CONTEXT: Trainees are exposed to medical errors throughout medical school and residency. Little is known about what facilitates and limits learning from these experiences.

OBJECTIVE: To identify major factors and areas of tension in trainees' learning from medical errors.

DESIGN, SETTING, AND PARTICIPANTS: Structured telephone interviews with 59 trainees (medical students and residents) from 1 academic medical center. Five authors reviewed transcripts of audiotaped interviews using content analysis.

RESULTS: Trainees were aware that medical errors occur from early in medical school. Many had an intense emotional response to the idea of committing errors in patient care. Students and residents noted variation and conflict in institutional recommendations and individual actions. Many expressed role confusion regarding whether and how to initiate discussion after errors occurred. Some noted the conflict between reporting errors to seniors who were responsible for their evaluation. Learners requested more open discussion of actual errors and faculty disclosure. No students or residents felt that they learned better from near misses than from actual errors, and many believed that they learned the most when harm was caused.

CONCLUSIONS: Trainees are aware of medical errors, but remaining tensions may limit learning. Institutions can immediately address variability in faculty response and local culture by disseminating clear, accessible algorithms to guide behavior when errors occur. Educators should develop longitudinal curricula that integrate actual cases and faculty disclosure. Future multi-institutional work should focus on identified themes such as teaching and learning in emotionally charged situations, learning from errors and near misses and balance between individual and systems responsibility.

KEY WORDS: medical errors; medical education; UME; GME; teaching methods.

DOI: $10.1111 / \mathrm{j} \cdot 1525-1497.2006 .00420 . \mathrm{x}$

J GEN INTERN MED 2006; 21:419-423.

$\mathrm{W}$ hile the prevalence of medical errors is controversial, ${ }^{1}$ it is well documented that errors occur at alarmingly high rates. ${ }^{2,3}$ Since the Institute of Medicine report To Err Is Human, the health care community has focused considerable attention on the reduction of medical error with a particular emphasis on quality improvement and systems changes. ${ }^{4-9}$ Studies have specifically noted patient ${ }^{10-12}$ and attending physician $^{12,13}$ responses and attitudes toward errors. Errors are part of both medical practice and training. ${ }^{14-16}$ The impor-

Address correspondence and requests for reprint to Dr. Fischer: University of Massachusetts Medical School, 55 Lake Avenue North, Benedict A3-118, Worcester, MA 01655 (e-mail: fischerm@ummhc.org). tance of addressing medical error in training programs has been reinforced by oversight organizations. ${ }^{14,17}$ Despite recognition of medical error as a critical component of medical education, there has been little study of how medical students and residents learn from and respond to medical errors.

One recent qualitative study focused on preceptors' responses to learners' errors, and identified themes for faculty development programs. ${ }^{13}$ Another characterized resident perceptions of the types and cause of errors. ${ }^{16}$ Others have described the Morbidity and Mortality conference, offering suggestion for more effective teaching. ${ }^{18}$ Decades ago Mizrahi $^{19}$ noted resident defenses (denial, discounting, and distancing) in response to errors, and $\mathrm{Wu}^{20}$ found that accepting responsibility for and discussing errors was associated with more constructive behavioral change. Twenty years later, we know little more about what trainees learn from medical errors and how to maximize learning to improve patient care. We designed this qualitative study to identify factors that affect learning from medical errors.

\section{METHODS}

All students enrolled in the University of Massachusetts Medical School (UMMS), and Internal Medicine and Surgery residency programs in 2003 to 2004 were invited to participate. Participants received a $\$ 25$ gift certificate for completing a single semistructured telephone interview. Of the 72 students who responded, $30(42 \%)$ were randomly selected for inclusion. All 29 residents who responded (21 IM postgraduate year (PGY) 1-3, 8 Surgery PGY 1-4) were included. Subjects provided written informed consent.

Semistructured interviews were conducted between October 2003 and January 2004. All interviewees were asked 7 questions regarding attitudes and response toward errors. Average interview length was approximately 25 minutes. Questions were generated from review of the literature, investigators' prior work, discussion with local faculty and educational leaders. While all participants were asked to discuss their responses to a specific error with which they had personal involvement, we offered a hypothetical case for those who could not recall such an event. This case described a student's order, cosigned by a resident for a medication to which the patient had well-documented allergy. The patient suffered anaphylaxis, required 3 days of ICU care, but recovered. The interview was pilot tested on 3 students not participating in the study. Questions were revised based on these experiences. Study interviews were conducted over the phone by a single author (J.B.) who participated in the pilot tests. 
Interviews were audiotaped, transcribed, and analyzed by 5 authors (M.F., K.M., E.A., D.D., M.P.) in an iterative process using content analysis. ${ }^{21,22}$ Reviewers were blinded to subject name. One author (M.F.) developed an initial coding key. Each author read 3 transcripts, identified, and coded major themes and important comments and noted additional topic areas. Findings were summarized by 1 author (M.F.) who revised the coding key. Authors reviewed the changes and the process was repeated using new transcripts until no new themes were identified. Two authors (M.F., K.M.) read all of the transcripts. This study was exploratory and not intended to identify numeric differences in responses by year or training program. Further work will address these quantitative issues. The study was approved by the UMMS Institutional Review Board for human subjects.

\section{RESULTS}

Major factors influencing learning from medical errors were identified in 4 topic areas: awareness of errors; factors influencing learner response (personality, hidden curriculum, event characteristics, role confusion, professional, or legal consequences); types of responses reported (emotional, cognitive, and behavioral-taking responsibility, disclosure, reflection); formal teaching.

\section{Awareness of Errors}

Regardless of level of training, nearly all students and residents said that they think about medical errors. They described errors as "inevitable" and "part of the practice of medicine." As 1 surgery resident said "I'm concerned every time [I] write an order-every time [I] do a procedure and ... when [I'm] on call." Another thought about errors "continually." Some expressed faith that the medical system would protect them from committing most errors.

Some students reported thinking about errors even before entering medical school, "I think its one of the scariest things in becoming a doctor, realizing how much responsibility you have and that human error happens all the time. [I thought about it] even before I [decided] ... I definitely wanted to go to medical school." Many learners said that their attitudes toward errors have changed during training. Even preclinical students reported shifts in their perspectives on medical errors, identifying more with providers than patients.

\section{Factors Influencing Learner Response}

Much of the teaching and learning around medical errors is informal. Among the factors that influence whether or how learning occurs are personality of the attending, hospital or program, the hidden curriculum (e.g., influences of organizational structure and culture on learning), ${ }^{23}$ characteristics of the event, role confusion, and professional or legal consequences.

Personality. Some learners felt that individual supervisor personality regulated response to error and was therefore important in their learning. Others noted the role of the program or institution. One resident reported seeing someone "trying to kind of cover [an error] up" at another hospital, and stated "I don't think I would have seen this at my own hospital because I think that people would have been more open." Even within an institution, students and residents experienced different responses to an error. One resident noted "even being on a different team within the same hospital, making a mistake can be looked at as an opportunity to learn versus an opportunity to chastise."

Hidden Curriculum. Some learners indicated that whatever their personal tendencies, they quickly assumed the "perspective of medicine' as they began training. "The more I get into the medical profession the more I kind of want to defend doctors in making mistakes." Most learners felt that the influence of this 'adopted' medical culture superceded their individual ethic. "In my mind I know what I think is the right thing to do, but sometimes it's a little different than culture dictates." Or as one student said, "part of the medical community does not want you to speak up about what you've done that was wrong. If I [apologized for making a serious error] there would be a number of people who would be upset at me for being too much like a bleeding heart and not enough of a tough professional and not being aware enough of the current litigious medical situation." Some noted that they have changed how they would respond since starting medical training, "in the past I've automatically thought of myself as somebody who's going to go and own up directly to the person, and maybe now I'm not as sure I would do that."

Event Characteristics. Specifics of an error, such as the history of the error and err-er, the severity of the patient injury, the proximate cause of the error and whether it was caught before it reached the patient (a near miss) also influenced how students and residents responded to and learned from medical errors. Many learners felt that they would learn better from errors they had made themselves. However, respondents also said open discussion of others' errors was effective for learning. Error characteristics such as cause (knowledge vs attitude), trainee's history of error-making, and accepting responsibility for an error, seemed most important in how learners felt that others would respond to them, which would impact their learning. As one student noted, "[if I] had done something maliciously wrong, then it would be different." Learners also felt that single errors were less likely to be viewed negatively than repeated errors or patterns of errors.

Most respondents stated they would learn better from actual errors than near misses. "You would still learn but it wouldn't be as complete learning as knowing about an error that has happened and what exactly the consequences were." Some noted they would learn best from errors that caused severe harm. This seemed to be based on the personal and emotional impact of such events. "If it has a poor outcome it's more likely to stick in my head because you feel, obviously, horrible for causing harm to the patient and causing a poor outcome, versus if there's no poor outcome, then you may be less likely to keep it in your mind." Unfortunately, some respondents questioned the longevity of learning even from errors with the worse outcomes "the problem is, it's kind of easy to fall back into being lax again."

Role Confusion. While many learners were certain of how they wanted to respond to errors, they were unsure of how this fit with their role in the team. This was more likely to be an issue with students than residents, and was particularly prevalent regarding error discussion and patient disclosure. Some noted that their own wishes were at odds with what they saw and 
were taught "I think it's important to talk to patients [about errors] ... but the problem is ... I feel like we're almost not supposed to."

Many learners acknowledged that their level of training played a role in their learning from errors. This was particularly true for the earlier, preclinical students, but was also reported by some senior residents. As the junior members of a team, learners noted tension that made it difficult for them to point out the errors of their seniors, or talk with those responsible for their evaluations. "I really had this problem which is that the only people who you can talk to about these things really ... are the same people who are grading you, and so it creates a very difficult dynamic because if you think that the team made a mistake and your resident doesn't want to talk about it, you really can't talk about it then."

Professional or Legal Consequences. Interviewees at all levels of training said that concern for potential legal consequences influenced their discussion and disclosure of errors. One resident admitted "I think everyone kind of worries about making that large error that's really going to cause injury or harm to a patient that may result in legal action." Some believed that having a close relationship with the patient would be protective against legal action. "We're taught in medical school one of the best ways to avoid malpractice is to have a very good working relationship with the patient." There were conflicting views on whether academic risk influenced trainee behavior. One student noted, "I'm more concerned about what I'm doing to the person that I'm treating than I am surrounding a grade . . . if I screw this up, what am I going to do to this person?"

\section{Types of Responses Reported}

Emotional Responses. Students and residents reported powerful emotional reactions to even thinking about committing errors. They used such words as "scared ... guilty," "embarrassed," "fear," "mad at myself ... extraordinarily awful," "frightening and discouraging," "afraid that I'd get in trouble with my attending" to describe their feelings. One resident reported thinking about errors because "being an internist is really scary. A lot of times I'm making decisions that I'm not $100 \%$ sure about, especially early on. You have to think about it because you're scared you're going to do something awful to somebody." Some noted personal professional consequences such as loss of self-confidence.

As noted above, many felt that these internal responses influenced their learning. One resident said sharing errors with others might decrease this anxiety, but continued, "I don't necessarily think that the level of anxiety should be lowered because I think it's an appropriate physiological response to something that's very important." Some respondents indicated that there were systems in place to debrief from errors, but that these were not utilized. "They say you can call for like a meeting of everyone who was there and talk about the codes ... I've never seen that done, even in horrific codes, I've never seen that done."

Cognitive and Behavioral Response. Learners' cognitive and behavioral responses fell into 3 broad categories: taking responsibility, disclosure, and reflection.
Taking Responsibility. Most learners felt it was important to take responsibility for errors. Some were nervous about discussing errors that they made. One student noted the importance of talking to the team and attending, but said "I think I would be sort of embarrassed to talk to the people on the team that I was working with." A resident also felt taking responsibility was important, but said "you don't want to lose respect among your peers." Many seemed to feel that this step was important to their learning, and perhaps a way to avoid negative consequences. "If you're sort of defending a mistake you made ... it takes away from your learning from it and it projects further onto others that you still feel like you weren't responsible or directly responsible, which is different from somebody who realizes that they've made a mistake and they're more than likely not to make the same mistake again."

Disclosure. Learners generally reported that talking to the patient or family was the "right" thing to do, especially for errors with noticeable consequences, however there were concerns about legal and professional ramifications as above. Although some felt open disclosure of errors reinforced patient trust, others discussed the difficulty of caring for patients after having made an obvious error in their care. "Now they're back in your care. That person has zero confidence in you. Your rapport is shot. Its very difficult to keep conducting patient care in that situation."

Many learners were unsure of whether to disclose errors to patients, who should be involved in disclosure conversations, and what to say. Many also felt uneasy about the conflict between their own beliefs and their training. "The thing I've been trained not to do is to apologize or admit wrongdoing to a patient, which is a very difficult thing for me because I'm very inclined to say I am at fault."

Reflection. When asked what they would do to avoid repeating errors, nearly all interviewees noted an element of reflection on their role. Some reported they would try to develop more systematic approaches to care. "It would encourage me to create a better system or protocol to follow to try to prevent something like that from happening." Others sought greater understanding of "why this happened," and improvement. "What is important, I think, is that after you do the mistake, and also when you do something good, reevaluate what you did and the changes that you can make. That's, I think, the most important thing."

\section{Formal Teaching}

Trainees experienced a variety of specific methods used to teach about medical error. These included lecture, Grand Rounds, orientation activities, morbidity and mortality conference, small group discussion, discussion of actual error cases, short courses, simulation/objective structured clinical examination (OSCE), e-mail communication, attending rounds discussion. Some indicated that they had had none despite standard trainee curriculum. When asked about what further teaching they would like, they suggested activities such as chart review and panel discussion. Many requested more small group discussions focusing on "real errors," and those presented by senior physicians who had committed them. This seemed to be important for support and learning. "I think there is some peace of mind hearing from practicing physicians, like if they're willing to open up and say, I've made errors. These are 
some of the examples of my errors. Not to say that its ok or a good thing to make an error, but just saying that ... you don't need to retire from the business because you've made 1 error or even a handful of errors. Its just a reminder that other people are doing it but we all need to continue to work on reducing the number of errors." They frequently noted that they were not aware of any clear protocol for what to do when an error occurred (beyond caring for the patient) and that such information would be very useful.

\section{DISCUSSION AND RECOMMENDATIONS}

Addressing Formal and Hidden Curricula. Trainees are keenly aware of the pervasiveness of medical errors. In many cases their attitudes and responses toward errors are changing very early in their training, as they start to embrace the culture of medicine. Learners may be reporting the effects of the hidden curriculum described earlier on medical errors. ${ }^{23-27}$ Recommendation: Our study suggests formal curricula should begin early in medical school to counter this effect. Faculty development will be required to facilitate open discussion with physicians at all levels of practice. Such initiatives may reshape medical culture and make explicit the currently hidden curriculum regarding medical errors, which is the first step in transformation. ${ }^{23-27}$ Because institutional and faculty responses are varied, individual interventions may be required to achieve maximum impact.

Faculty and Patient Case Conferences and Other Suggestions for Teaching. Regarding formal training, learners reported many techniques used in teaching about medical errors. Trainees at all levels want more open discussion of actual errors. Recommendation: While further study is warranted, our findings suggest that actual cases of faculty members who have committed errors, and including patients who have been the subject of errors, are likely to be effective in addressing both physician and patient responses. We report suggestions for teaching (Table 1).

Development and Dissemination of Local Policy. Medicine still practices a modified apprenticeship. Even senior trainees felt some confusion about their nonclinical role if an error occurred. The conflict of reporting errors made by one's seniors or evaluators is particularly important. Recommendation: Training hospitals and schools should develop clear, accessible guidelines to direct response to errors at all levels of training in order to alleviate this conflict, address concerns about legal liability and ramifications on trainee evaluation.

Impact of Emotions on Teaching and Learning. Teaching and learning from medical errors differs from that around other clinical experiences in part because of the emotional impact of the error. Learners report significant stress as a result of even imagining committing medical errors. The fear and selfdoubt reported by learners is consistent with preceptor views. ${ }^{13}$ However, we have little specific knowledge of how these emotions effect learning, whether increased publication of errors may cause "desensitization" or how to direct learning in such emotionally charged situations. ${ }^{28,29}$ Recommendation: Further work should determine how to ensure both learning and support while addressing the emotional impact of error commission.

Learning from Errors, Near Misses, Peers, and Simulation. Trainees believe they learn more from their own errors, and those that cause harm to patients. This may be related to increased emotional impact of such events. While these factors are not mutable, it is important to understand their individual contribution to learning in order to shape curricular response. Recommendation: Use of simulation may help to understand and bridge this experiential gap. Further research should explore learning from near misses and others' errors. ${ }^{30}$

Balance of Individual and Systems Responsibility. Learners' opinions are consistent with Wu's findings that those who accept responsibility for an error are most likely to make a constructive change in response to it. ${ }^{20}$ Many proponents of patient safety emphasize system causes for and solutions to errors. This may interfere with learners' taking responsibility for their role in errors, which may limit the degree they learn from their mistakes and diminish the degree to which they take constructive action. Recommendation: The balance of individual and systems responsibility, and the effects of sys-

Table 1. Author Recommendations for Training

\begin{tabular}{lll}
\hline \hline Venue & Method \\
\hline
\end{tabular}

Formal teaching reported by

trainees

Grand rounds, core lecture

series

Small groups

Simulation

Short courses

Interdisciplinary rounds

E-mail

Attending rounds

Other teaching methods

recommended by trainees

Chart review

Panel discussion
Interactive lecture

Team learning, case-based learning OSCE

Interactive didactic, modeling, faculty development Case discussion

Directive

Modeling, case discussion
Background, policy dissemination

Individual error discussions

Error discussion in teams, disclosure to patients

Policy, root cause analysis, disclosure technique

Common errors seen in pharmacy, allied health fields, root cause analysis

Policy, banned abbreviation

Addressing commonality of errors, specific knowledge and skills, emotional support
Practice-based learning and improvement Modeling
Quality improvement, case discussion

Common errors, approach to response, psychological readiness

OSCE, objective structured clinical examination. 
tems accountability on individual learning should be studied.

This study has a number of potential limitations. Subjects were interviewed over the phone, but may not have felt comfortable responding honestly. Although we sought personal experiences with specific error events, in many cases respondents preferred discussing the hypothetical case. All subjects were from a single institution, but many had training experiences at other institutions and discussed those during the interviews. We chose to interview only students and residents as they have been underrepresented in prior studies and offer significant opportunities for educational intervention. Further information could be obtained from other members of the health care team including nurses, fellows or allied health professionals. Finally, as is true in any qualitative research effort, author bias may have played a role in how factors were identified and categorized.

\section{CONCLUSION}

Our findings suggest that trainees are keenly aware of medical errors. We have identified a number of factors that influence how trainees respond to and learn from medical errors. Institutions can immediately address individual variability in faculty response and local culture by disseminating clear, accessible response algorithms to guide behavior when errors occur. Educators should develop longitudinal curricula that utilize a variety of teaching methods, integrate actual cases and faculty disclosure to address both trainee knowledge and the hidden curriculum. Future multi-institutional work should focus on major themes identified such as teaching and learning in emotionally charged situations, learning from near misses as well as errors that cause patient harm, and appropriate levels of individual and systems responsibility.

Thanks to Mark D. Schleinitz, MD, MSc, for his thoughtful feedback. This work was supported by a grant from the Meyers Primary Care Institute. The granting agency had no role in the design, conduct, analysis, report, or review of the manuscript beyond specific roles of identified authors.

\section{REFERENCE}

1. Hayward RA, Hofer TP. Estimating hospital deaths due to medical errors: preventability is in the eye of the reviewer. JAMA. 2001;286:415-20.

2. Institute of Medicine. To Err Is Human. Washington, DC; National Academy Press; 1999.

3. Vincent C, Neale G, Woloshynowych M. Adverse events in British hospitals: preliminary retrospective record review. BMJ. 2001;322:517-9.

4. Braithwaite RS, DeVita MA, Mahidhara R, Simmons RL, Stuart S, Foraida M. Medical Emergency Response Improvement Team (MERIT). Use of medical emergency team (MET) responses to detect medical errors. Qual Safety Health Care. 2004;13:255-9.

5. Classen DC, Kilbridge PM. The roles and responsibility of physicians to improve patient safety within health care delivery systems. Acad Med. 2002;77:963-72.
6. Ziegelstein RC, Fiebach NH. "The mirror" and "the village": a new method for teaching practice-based learning and improvement and systemsbased Practice. Acad Med. 2004;79:83-8.

7. Institute of Medicine. Crossing the Quality Chasm: The IOM Health Care Quality Initiative. Washington, DC; National Academy Press; 2001.

8. Institute of Medicine. Health Professions Education: A Bridge to Quality. Washington, DC; National Academy Press; 2003.

9. Liang BA. A system of medical error disclosure. Qual Safety Health Care. 2002;11:64-8.

10. Mazor KM, Simon SR, Yood RA, et al. Health plan members' views about disclosure of medical errors. Ann Intern Med. 2004 Mar 16;140:409-18.

11. Mazor KM, Simon SR, Yood RA, et al. Health plan members' views on forgiving medical errors. Am J Managed Care. 2005; 11:49-52.

12. Gallagher TH, Waterman AD, Ebers AG, Fraser VJ, Levinson W. Patients' and physicians' attitudes regarding the disclosure of medical errors. JAMA. 2003;289:1001-7.

13. Mazor KM, Fischer MA, Haley H-L, Hatem D, guirk ME. Teaching and learning around medical errors: primary care preceptors' views. Med Educ. 39:982-90.

14. Patient Safety and Graduate Medical Education. AAMC Report from the Joint Committee of the Group on Resident Affairs and Organization of Resident Representatives. February 2003.

15. Volpp KG, Grande MD. Residents' suggestions for reducing errors in teaching hospitals. N Engl J Med. 2003;348:851-5.

16. Schenkel S, Khare R, Rosenthal M, Sutcliffe K, Lewton E. Resident perceptions of medical errors in the emergency department. Acad Emerg Med. 2003;10:1218-324.

17. ACGME Outcomes Project. www.ACGME.org/Outcomes.

18. Pierluissi E, Fischer MA, Campbell AR, Landefeld CS. Discussion of medical errors in morbidity and mortality conferences. JAMA. 2003;290:2838-42.

19. Mizrahi T. "Managing medical mistakes: ideology, insularity and accountability among internists-in-training." Soc Sci Med. 1984;19: 135-46.

20. Wu AW, Folkman S, McPhee SJ, Lo B. Do house officers learn from their mistakes? JAMA. 1991;265:2089-94.

21. Krueger RA, Casey MA. Focus Groups. 3rd edn. Thousand Oaks, CA: Sage Publications; 2000.

22. Miles M, Huberman AM. Qualitative Data Analysis. 2nd edn. Thousand Oaks, CA: Sage Publications; 1994.

23. Hafferty FW. Beyond curriculum reform: confronting medicine's hidden curriculum. Acad Med. 1998;73:403-7.

24. Thiedke C, Blue AV, Chessman AW, Keller AH, Mallin R. Student observations and ratings of preceptor's interactions with patients: the hidden curriculum. Teach Learn Med. 2004;16:312-6.

25. Lempp H, Seale $\mathbf{C}$. The hidden curriculum in undergraduate medical education: qualitative study of medical students' perceptions of teaching. BMJ. 2004;329:770-3.

26. Hundert EM, Hafferty F, Christakis D. Characteristics of the informal curriculum and trainees' ethical choices. Acad Med. 1996;71: 624-42.

27. Stern D. Practicing what we preach? An analysis of the curriculum of values in medical education. Am J Med. 1998;104:569-75.

28. Burack JH, Irby DM, Carline JD, Root RK, Larson EB. Teaching compassion and respect. Attending physicians' responses to problematic behaviors. J Gen Intern Med. 1999; 14:49-55.

29. Ende J, Pomerantz A, Erickson F. Preceptors' strategies for correcting residents in an ambulatory care medicine setting: a qualitative analysis. Acad Med. 1995;70:224-9.

30. McCafferty MH, Polk HC Jr. Addition of "near-miss" cases enhances a quality improvement conference. Arch Surg. 2004;139:216-7. 